\title{
ESTRATEGIA PARA REDUCIR INTERVALOS DE INCERTIDUMBRE APLICADA EN LOCALIZACIÓN DE FALLAS EN SISTEMAS DE DISTRIBUCIÓN
}

\author{
STRATEGY TO REDUCE THE UNCERTAINTY INTERVALS APPLIED IN FAULT LOCATION FOR \\ POWER DISTRIBUTION SYSTEMS
}

César Augusto Orozco Henao

Ing. Eléctrico, M.Sc. Investigador, Universidad Tecnológica de Pereira. Colombia caorozco@utp.edu.co

Juan José Mora Flórez

Ing. Eléctrico, PhD, Profesor asociado, Universidad Tecnológica de Pereira. Colombia jjmora@utp.edu.co.

Sandra Milena Pérez Londoño

Ing. Eléctrico, Ph.D.(c), Profesora titular, Universidad Tecnológica de Pereira. Colombia saperez@utp.edu.co.

Fecha de recepción: 22 de Abril de 2012

Fecha de aprobación: 1 de diciembre 2012

\section{RESUMEN}

El método de localización propuesto en este artículo se basa en una estrategia iterativa que determina el valor mínimo de la reactancia de falla, utilizando la técnica de reducción de intervalos de incertidumbre de Fibonacci. Esta técnica mejora el método de localización de fallas debido a que aumenta su precisión, mediante la aplicación de un tamaño de paso variable para determinar el valor absoluto de la reactancia de falla. La estrategia utiliza las mediciones de tensión y corriente en la subestación, por lo tanto, considera las variaciones en los estados de pre-falla y falla. El método se valida en el sistema de prueba IEEE 34 nodos conformado por laterales monofásicos, bifásicos y trifásicos, para todos los tipos de fallas y considerando resistencias de falla entre 0 y $40 \mathrm{ohms}$. Los resultados obtenidos muestran errores de estimación inferiores al 1,8\%, lo cual confirma la validez de la propuesta.

Palabras clave: localización de fallas, técnicas de reducción de intervalos de incertidumbre, métodos basados en la estimación de la impedancia, sistemas de distribución.

\begin{abstract}
In this paper, a strategy based on an iterative algorithm used to determine the minimum fault reactance, applied to reduce the Fibonacci uncertainty intervals is proposed. This strategy increases
\end{abstract}


the accuracy of the proposed fault location using a variable step to calculate the minimum fault reactance. The algorithm uses measurements of voltages and currents at the power substation also considering variations between pre-fault and fault steady states. The strategy is validated at the IEEE 34 test feeder, considering single-phase, two-phase and three-phase faults and also fault resistances from 0 to 40 ohms. According to the obtained results, the estimation error is lower than 1.8\%, which validates the good performance of the proposed approach.

Keywords: Fault location, uncertainty intervals, impedance-based method, power distribution systems.

\section{INTRODUCCIÓN}

Desde la reestructuración del sector eléctrico, la manera de evaluar el servicio de energía eléctrica cambió significativamente y se introduce un nuevo concepto conocido como calidad del servicio. Este concepto está asociado directamente a la continuidad de suministro, lo cual introduce diversos índices que permiten su cuantificación [1]. En Colombia, los índices más utilizados son: el índice de referencia agrupado de la discontinuidad (IRAD), y el índice trimestral agrupado de la discontinuidad (ITAD) [2].

La localización de fallas paralelas en sistemas de distribución es una temática asociada al desarrollo de herramientas que permitan mejorar la continuidad del servicio, con la disminución del tiempo de detección de zonas con alta probabilidad de falla, así como la suplencia temporal de algunas cargas que se ubican en el circuito fallado.

En la actualidad, se han desarrollado diferentes metodologías que utilizan el concepto de la determinación de la reactancia mínima de falla para localizar fallas en sistemas de distribución.

En Morales-España et al. [3], se presenta una propuesta basada en el principio de la reactancia mínima, que utiliza los parámetros de fase para determinar una expresión que define la reactancia de falla en función de la distancia al punto falla. Esta estrategia presenta varias características que pueden afectar su desempeño tales como: a) la concentración de la carga al final del circuito y b) la precisión depende del tamaño de paso que se utiliza para determinar la reactancia de falla. La última característica enfrenta la precisión del método de localización contra su esfuerzo computacional.

A partir del análisis de las desventajas presentadas anteriormente, Morales-España et al. [3], proponen algunas alternativas de mitigación en Morales-España et al. [4], donde determinan la distancia a la falla en función de la longitud del sistema y las reactancias de falla de secuencia positiva y negativa. De esta manera, logran obtener un método un poco más preciso pero no presenta con claridad, una técnica para considerar los intervalos de estimación de la reactancia. 
Existen otros métodos de localización de fallas que utilizan las mismas aproximaciones, tal como se presenta en [5], que según sus autores, alcanzan buenos resultados para las pruebas propuestas. Sin embargo, se siguen utilizando aproximaciones como la concentración de la carga al final de circuito, las cuales se pueden mejorar, si diseñan estrategias que permitan modelar la carga en cada sección del sistema.

Todas las metodologías referenciadas previamente en este artículo, utilizan un tamaño de paso constante para graficar la reactancia de falla y determinar la localización de la misma. Para la propuesta aquí presentada, se diseña una estrategia que utiliza un modelado de carga en cada sección y una técnica de reducción de intervalos de incertidumbre de Fibonacci, que introduce un tamaño de paso variable para determinar el mínimo valor de la reactancia de falla y así mejorar la estimación de la falla.

Como contenido, en la sección 2 de este artículo, se presenta el desarrollo matemático para estimar la reactancia de falla en un sistema de potencia. En la sección 3, se presenta la técnica de reducción de intervalos de incertidumbre de Fibonacci; mientras que en la sección 4, se presenta la estrategia de localización de fallas propuesta. La sección 5 está dedicada a la presentación de las pruebas y los resultados obtenidos para un sistema de distribución prototipo. Por último, en la sección 6, se presentan las conclusiones más importantes asociadas a esta investigación.

\section{ESTIMACIÓN DE LA REACTANCIA DE FALLA EN UN SISTEMA DE POTENCIA}

Para este análisis, se asume un alimentador de distribución que tiene una falla entre el nodo k y el k+1, como el mostrado en la Figura 1. En este alimentador, sólo se tiene medidas de tensión y corriente en la subestación [7].

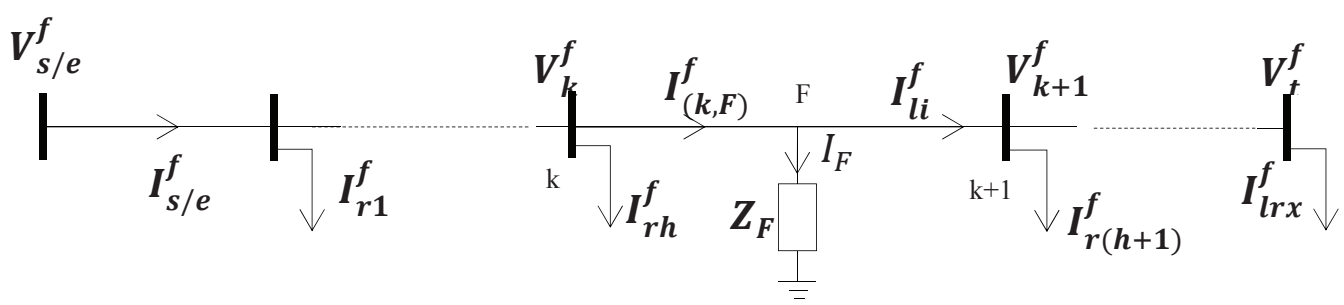

Figura 1. Modelo unifilar de una falla en un alimentador de distribución.

Donde, las variables ante el estado de falla, indicadas con el superíndice f son:

$\boldsymbol{V}_{\mathbf{c} / \boldsymbol{e}}^{\boldsymbol{f}}$ : Tensión en la subestación

$\boldsymbol{I}_{\mathbf{c} / \boldsymbol{e}}^{\boldsymbol{f}}$ : Corriente en la subestación

$\boldsymbol{V}_{\boldsymbol{k}}^{\boldsymbol{f}}$ : Tensión en el nodo $\mathrm{k}$ 
$\boldsymbol{I}_{(\boldsymbol{k}, \boldsymbol{F})}^{\boldsymbol{f}}$ : Corriente de fase hacia la sección en falla

$\boldsymbol{I}_{\boldsymbol{l i}}^{\boldsymbol{f}}$ : Corriente de carga para la fase i (i=a, b, c)

$\boldsymbol{I}_{\boldsymbol{r} \boldsymbol{h}}^{\boldsymbol{f}}$ : Corriente que se deriva por el lateral $\mathrm{h}$

$\boldsymbol{I}_{\boldsymbol{F}}$ : Corriente por la impedancia de falla $\left(Z_{\mathrm{F}}\right)$

A partir de los registros de tensión y corriente en la subestación, se calcula la tensión y la corriente para cada tramo que conforma el alimentador [8]. Despues, se puede reducir el análisis de cortocircuito exclusivamente al tramo donde ocurrió la falla, tal como se muestra en esta sección [7].

El método se fundamenta en la determinación de la mínima reactancia de falla, tal como se propone en [3]. En la sección bajo análisis, se considera que la impedancia es puramente resistiva en el nodo de falla.

\subsection{ANÁLISIS PARA FALLA MONOFÁSICA}

Se asume la falla monofásica modelada como se muestra en la Figura 2, para la sección de interés entre el nodo ky $\mathrm{k}+1$.

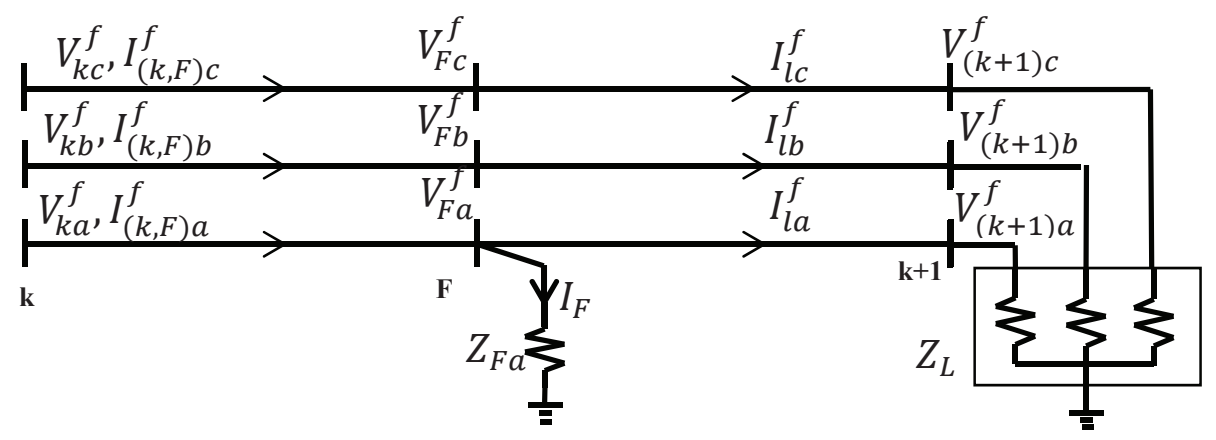

Figura 2. Modelo equivalente de una falla monofásica.

A partir del análisis circuital del modelo mostrado en la Figura 2, se obtiene el sistema de ecuaciones (1).

$$
\left[\begin{array}{c}
V_{F a}^{f} \\
V_{F b}^{f} \\
V_{F c}^{f}
\end{array}\right]=\left[\begin{array}{c}
V_{k a}^{f} \\
V_{k b}^{f} \\
V_{k c}^{f}
\end{array}\right]-\left[\begin{array}{ccc}
m Z_{a a} & m Z_{a b} & m Z_{a c} \\
m Z_{b a} & Z_{b b}+Z_{l b} & Z_{b c} \\
m Z_{c a} & Z_{c b} & Z_{c c}+Z_{l c}
\end{array}\right]\left[\begin{array}{c}
I_{(k, F) a}^{f} \\
I_{(k, F) b}^{f} \\
I_{(k, F) c}^{f}
\end{array}\right]
$$


Donde:

$\boldsymbol{I}_{(\boldsymbol{k}, \boldsymbol{F}) \boldsymbol{i}}^{\boldsymbol{f}}$ : Corriente de la fase i en la sección en falla

$\boldsymbol{V}_{\boldsymbol{k} \boldsymbol{i}}^{\boldsymbol{f}}$ : Tensión de la fase i en el nodo $\mathrm{k}$

$m$ : Distancia a la falla en por unidad de longitud

$\boldsymbol{Z}_{\boldsymbol{l i}}$ : Impedancia de carga concentrada en el nodo $\mathrm{k}+1$ para la fase i

$Z_{i i}$ : Impedancia propia de la línea para la fase i, en la sección en falla

$Z_{i j}$ : Impedancia mutua de la línea entre fases i y j, en el tramo en falla $(i \neq j)$

$\boldsymbol{V}_{\boldsymbol{F} \boldsymbol{i}}^{\boldsymbol{f}}$ : Tensión de la fase i en el nodo de falla

De manera similar, se define una expresión para la corriente de falla $\left(I_{F i}\right)$, en función de la distancia a la falla $(m)$.

$$
\left[\begin{array}{l}
I_{F a} \\
I_{F b} \\
I_{F C}
\end{array}\right]=\left[\begin{array}{c}
I_{(k, F) a}^{f} \\
I_{(k, F) b}^{f} \\
I_{(k, F) c}^{f}
\end{array}\right]-\left[\begin{array}{c}
I_{l a}^{f} \\
I_{l b}^{f} \\
I_{l c}^{f}
\end{array}\right]
$$

Donde $I_{l}^{f}$, es la corriente de carga en el estado de falla y se define por la ecuación (3).

$$
\left[\begin{array}{c}
I_{l a}^{f} \\
I_{l b}^{f} \\
I_{l c}^{f}
\end{array}\right]=\left(\left[\begin{array}{ccc}
(1-m) Z_{a a} & (1-m) Z_{a b} & (1-m) Z_{a c} \\
(1-m) Z_{b a} & Z_{b b}+Z_{l b} & Z_{b c} \\
(1-m) Z_{c a} & Z_{c b} & Z_{c c}+Z_{l c}
\end{array}\right]-\left[\begin{array}{ccc}
Z_{l a} & 0 & 0 \\
0 & Z_{l b} & 0 \\
0 & 0 & Z_{l c}
\end{array}\right]\right)^{-1}\left[\begin{array}{c}
V_{F a}^{f} \\
V_{F b}^{f} \\
V_{F c}^{f}
\end{array}\right]
$$

Una vez se estima la tensión en el nodo de falla $V_{F}^{f}$ y la corriente de falla $I_{l}^{f}$, se puede determinar la impedancia de falla. A partir de la componente imaginaria, se obtiene la reactancia de falla en función de la distancia a la misma.

Este análisis se puede extender para fallas monofásicas en las fases b y c, mediante un cambio de simetría. El sistema de ecuaciones (4) representa una generalización de la estimación de la reactancia de falla para los tipos de falla monofásica. 


$$
\left[\begin{array}{c}
X_{F a}(m) \\
X_{F b}(m) \\
X_{F c}(m)
\end{array}\right]=\left[\begin{array}{ccc}
\operatorname{Imag}\left(\frac{V_{F a}^{f}(m)}{I_{F a}(m)}\right) & 0 & 0 \\
0 & \operatorname{Imag}\left(\frac{V_{F b}^{f}(m)}{I_{F b}(m)}\right) & 0 \\
0 & 0 & \operatorname{Imag}\left(\frac{V_{F c}^{f}(m)}{I_{F c}(m)}\right)
\end{array}\right]
$$

\subsection{ANÁLISIS PARA FALLA BIFÁSICA}

Se asume la falla bifásica entre los nodos $\mathrm{k}$ y k +1, modelada como se muestra en la Figura 3.

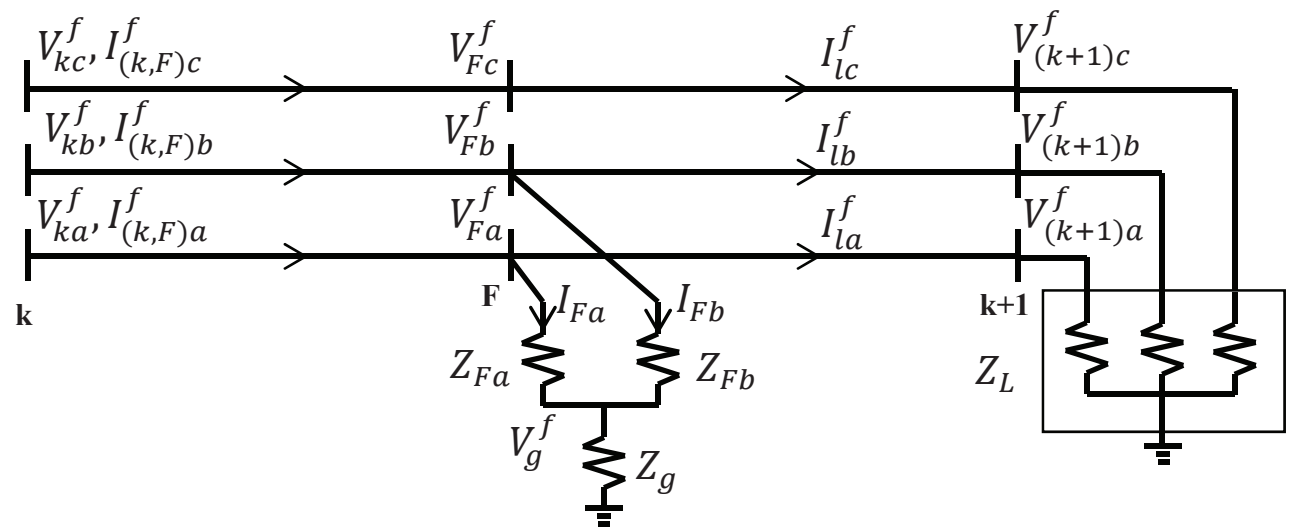

Figura 3. Modelo equivalente de una falla bifásica en un alimentador de distribución.

Al igual que para la falla monofásica, se realiza un análisis del circuito de la Figura 3, en función de la tensión en la resistencia del suelo $\left(V_{g}^{f}\right)$, con el fin de formular un análisis general para las fallas bifásicas y bifásicas a tierra. El sistema de ecuaciones del modelo general se presenta en (5).

$$
\left[\begin{array}{c}
V_{F a}^{f} \\
V_{F b}^{f} \\
V_{F c}^{f}
\end{array}\right]=\left[\begin{array}{c}
V_{k a}^{f}-V_{g}^{f} \\
V_{k b}^{f}-V_{g}^{f} \\
V_{k c}^{f}
\end{array}\right]-\left[\begin{array}{ccc}
m Z_{a a} & m Z_{a b} & m Z_{a c} \\
m Z_{b a} & m Z_{b b} & m Z_{b c} \\
m Z_{c a} & m Z_{c b} & Z_{c c}+Z_{l c}
\end{array}\right]\left[\begin{array}{c}
I_{(k, F) a}^{f} \\
I_{(k, F) b}^{f} \\
I_{(k, F) c}^{f}
\end{array}\right]
$$


La estimación de la corriente de falla se realiza, utilizando el sistema de ecuaciones (2). La corriente de carga en estado de falla, se determina por el sistema de ecuaciones (6).

$$
\left[\begin{array}{c}
I_{l a}^{f} \\
I_{l b}^{f} \\
I_{l c}^{f}
\end{array}\right]=\left(\left[\begin{array}{ccc}
(1-m) Z_{a a} & (1-m) Z_{a b} & (1-m) Z_{a c} \\
(1-m) Z_{b a} & (1-m) Z_{b b} & (1-m) Z_{b c} \\
(1-m) Z_{c a} & (1-m) Z_{c b} & Z_{c c}+Z_{l c}
\end{array}\right]-\left[\begin{array}{ccc}
Z_{l a} & 0 & 0 \\
0 & Z_{l b} & 0 \\
0 & 0 & Z_{l c}
\end{array}\right]\right)^{-1}\left[\begin{array}{c}
V_{F a}^{f} \\
V_{F b}^{f} \\
V_{F c}^{f}
\end{array}\right]
$$

Debido a que se presenta una nueva incógnita introducida por la tensión de la resistencia del suelo, no se puede determinar la impedancia de falla como la relación directa entre la tensión en el punto de falla y la corriente de falla. Una manera de estimar la impedancia de falla para cada fase, es restando las tensiones en los nodos fallados para eliminar la incógnita de la tensión de la resistencia del suelo.

Se debe tener especial cuidado, porque se asume que las impedancias de falla son iguales para cada fase fallada. Sin embargo, esta aproximación es adecuada, debido a que se ha observado en la práctica, que la resistencia de falla en los sistemas de distribución de energía bajo condición de una falla bifásica a tierra, es relativamente pequeña y con valores muy similares. Teniendo en cuenta lo anterior, se realiza el cambio de simetría para determinar una expresión general para la reactancia de falla bifásica y bifásica a tierra, tal como se presenta en (7).

$$
\left[\begin{array}{c}
X_{F a}(m) \\
X_{F b}(m) \\
X_{F c}(m)
\end{array}\right]=\left[\begin{array}{ccc}
\operatorname{Imag}\left(\frac{\Delta V_{a b}^{f}(m)}{I_{F a}(m)}\right) & 0 & 0 \\
0 & \operatorname{Imag}\left(\frac{\Delta V_{b c}^{f}(m)}{I_{F b}(m)}\right) & 0 \\
0 & \operatorname{Imag}\left(\frac{\Delta V_{c a}^{f}(m)}{I_{F c}(m)}\right)
\end{array}\right]
$$

Donde $\Delta V_{i j}^{f}$ es la diferencia entre las tensiones en el nodo de falla de las fases falladas $\left(\left(V_{F i}^{f}(m)-V_{F j}^{f}(m) \forall i \neq j\right)\right)$.

\subsection{ANÁLISIS PARA FALLA TRIFÁSICA}

Se considera una falla trifásica entre los nodos k y k +1 modelada como se muestra en la Figura 4. 


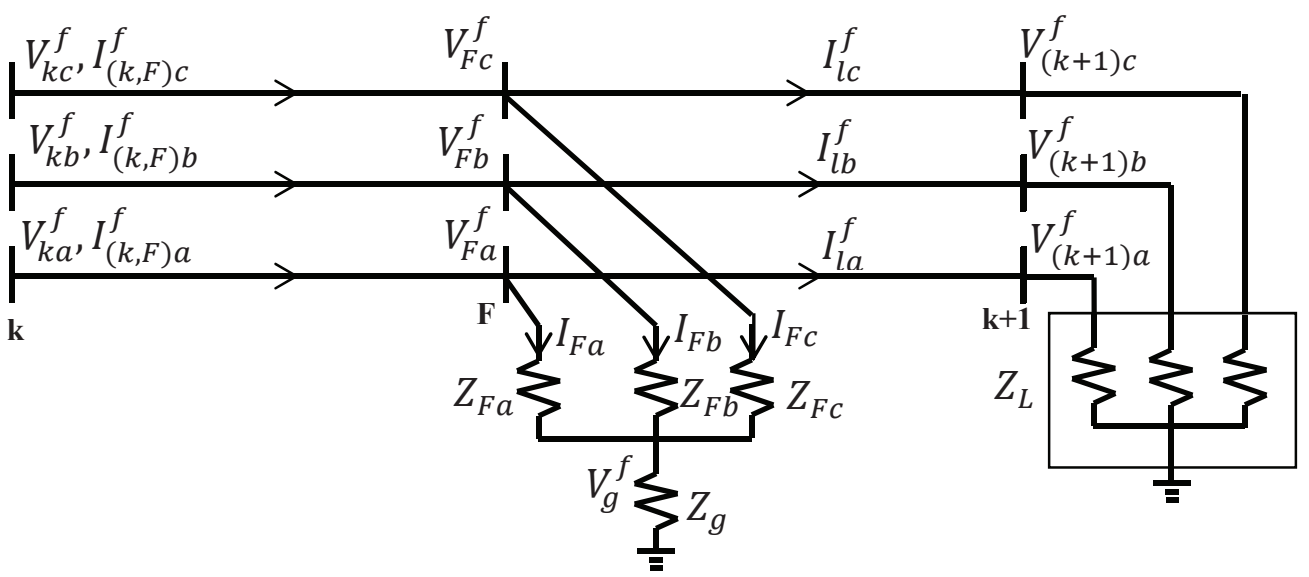

Figura 4. Modelo equivalente de una falla trifásica en un alimentador de distribución

Al igual que para los casos de fallas anteriormente mencionados, se requiere un sistema de ecuaciones para representar de manera exacta y general los dos tipos de fallas trifásicas (trifásicas y trifásicas a tierra). Por esta razón, el análisis se realiza en función de la tensión de la resistencia del suelo $\left(V_{g}^{f}\right)$, como se muestra en el sistema de ecuaciones (8).

$$
\left[\begin{array}{c}
V_{F a}^{f} \\
V_{F b}^{f} \\
V_{F c}^{f}
\end{array}\right]=\left[\begin{array}{c}
V_{k a}^{f}-V_{g}^{f} \\
V_{k b}^{f}-V_{g}^{f} \\
V_{k c}^{f}-V_{g}^{f}
\end{array}\right]-\left[\begin{array}{lll}
m Z_{a a} & m Z_{a b} & m Z_{a c} \\
m Z_{b a} & m Z_{b b} & m Z_{b c} \\
m Z_{c a} & m Z_{c b} & m Z_{c c}
\end{array}\right]\left[\begin{array}{c}
I_{(k, F) a}^{f} \\
I_{(k, F) b}^{f} \\
I_{(k, F) c}^{f}
\end{array}\right]
$$

Utilizando el sistema de ecuaciones (2) y (9), se estima la corriente de falla por las tres fases falladas.

$$
\left[\begin{array}{c}
I_{l a}^{f} \\
I_{l b}^{f} \\
I_{l c}^{f}
\end{array}\right]=\left(\left[\begin{array}{lll}
(1-m) Z_{a a} & (1-m) Z_{a b} & (1-m) Z_{a c} \\
(1-m) Z_{b a} & (1-m) Z_{b b} & (1-m) Z_{b c} \\
(1-m) Z_{c a} & (1-m) Z_{c b} & (1-m) Z_{c c}
\end{array}\right]-\left[\begin{array}{ccc}
Z_{l a} & 0 & 0 \\
0 & Z_{l b} & 0 \\
0 & 0 & Z_{l c}
\end{array}\right]\right)^{-1}\left[\begin{array}{c}
V_{F a}^{f} \\
V_{F b}^{f} \\
V_{F c}^{f}
\end{array}\right]
$$

De la misma manera que para la falla bifásica, la reactancia de falla para cada fase fallada, se determinada por la diferencia de las tensiones entre los nodos en falla, dividido entre la corriente de falla de la fase en estudio. El sistema de ecuaciones (10), representa la reactancia de falla para una falla trifásica y trifásica a tierra. 


$$
\left[\begin{array}{c}
X_{F a}(m) \\
X_{F b}(m) \\
X_{F c}(m)
\end{array}\right]=\left[\begin{array}{ccc}
\operatorname{Imag}\left(\frac{\Delta V_{a b}^{f}(m)}{I_{F a}(m)}\right) & 0 & 0 \\
0 & \operatorname{Imag}\left(\frac{\Delta V_{b c}^{f}(m)}{I_{F b}(m)}\right) & 0 \\
0 & \operatorname{Imag}\left(\frac{\Delta V_{c a}^{f}(m)}{I_{F c}(m)}\right)
\end{array}\right]
$$

Después de obtener las reactancias de falla del sistema de ecuaciones (10), se define la reactancia de falla, como el promedio aritmético de las tres, menos la desviación estándar, para corregir los problemas de sobrestimación de la distancia a la falla.

$$
\begin{array}{r}
\overline{X_{F}}=\frac{\sum_{i=a}^{c} X_{F i}}{3} \\
s=\sqrt{\frac{\sum_{i=a}^{c}\left(X_{F i}-\overline{X_{F}}\right)^{2}}{3}} \\
X_{F}=\overline{X_{F}}-s
\end{array}
$$

\section{ANÁLISIS DE INCERTIDUMBRE MEDIANTE LA TÉCNICA DE FIBONACCI}

El problema de localización de fallas se plantea como la minimización del valor absoluto de la reactancia de falla en función de la distancia a la falla $\left|X_{f}(m)\right|$. Esta función se define en el intervalo $[0,1]$, ya que $m$ se normaliza y el análisis se aplica a cada sección que conforma el sistema de potencia [3]. El intervalo es conocido como intervalo de incertidumbre, y el problema genérico para localización de fallas se modela en (14).

$$
\begin{aligned}
& \text { Minimizar }\left|X_{f}(\lambda, \mu)\right| \\
& \text { Sujeto a: } \\
& 0<\lambda<1 \\
& 0<\mu<1
\end{aligned}
$$

Este problema se resuelve por el método de búsqueda unidimensional de reducción de intervalos de Fibonacci. Se debe garantizar que la función $\left|X_{f}(m)\right|$ sea cuasi-convexa en el intervalo [0, 1], para asegurar que la solución determinada por la técnica corresponda al óptimo global de la 
función. Por fortuna, cuando se modela la reactancia de falla en una sección del sistema, el valor absoluto de esta función cumple con la condición de convexidad [9].

La estrategia de reducir los intervalos de Fibonacci se fundamenta en la reducción del intervalo de incertidumbre para funciones $\theta(\lambda): \mathfrak{R} \rightarrow \mathfrak{R}$, estrictamente cuasi-convexas. La reducción del intervalo de incertidumbre se realiza por medio de la definición de dos valores $\lambda$ y $\mu$, en función de la secuencia de números de Fibonacci, donde $\lambda, \mu \in[a, b]$ y, son valores en $\mathfrak{R}$ tales que $\lambda<\mu$. Para el problema de localización de fallas, $\lambda$ y $\mu$ pertenecen al intervalo [0, 1], y representan la variación de m sobre la sección en análisis, tal como se definen en las ecuaciones (15) y (16).

$$
\begin{aligned}
& \lambda_{q}=a_{q}+\frac{W_{n-q-1}}{W_{n-q+1}}\left(b_{q}-a_{q}\right) \\
& \mu_{q}=a_{q}+\frac{W_{n-q}}{W_{n-q+1}}\left(b_{q}-a_{q}\right)
\end{aligned}
$$

Donde:

$\lambda_{q}$ : Valor del parámetro $\lambda$ en la iteración q

$\mu_{q}$ : Valor del parámetro $\mu$ en la iteración q

$a_{q}$ : Extremo inferior del intervalo de incertidumbre de la iteración q

$b_{q}$ : Extremo superior del intervalo de incertidumbre de la iteración q

$W_{i}$ : i-ésimo número de la serie Fibonacci

n: Cantidad de números de Fibonacci por utilizar

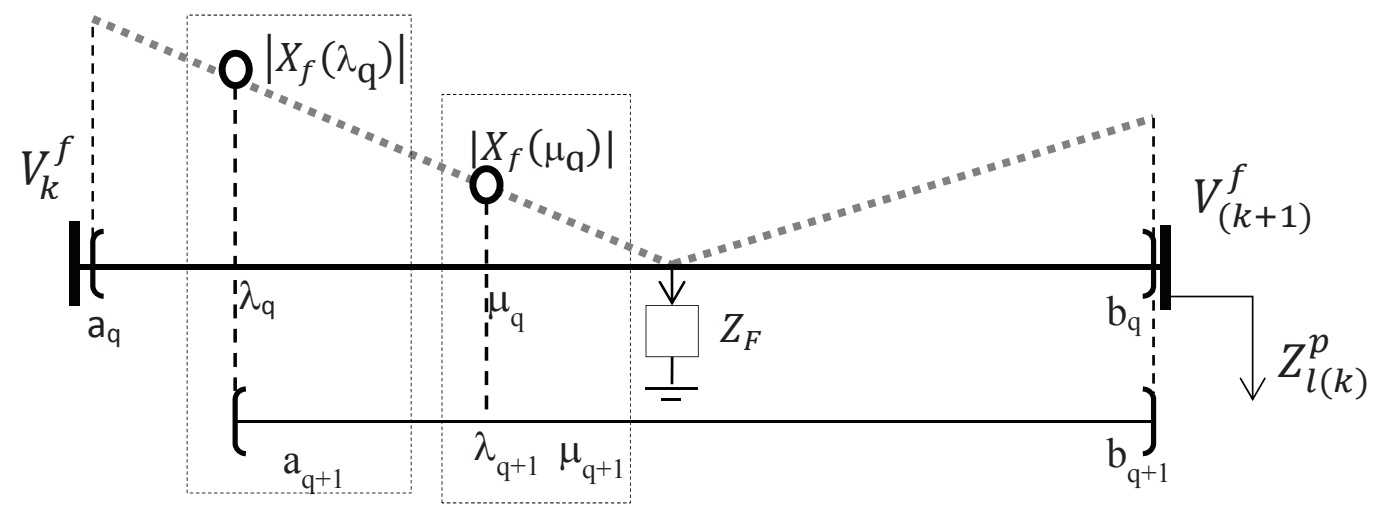

Figura 5. Determinación de nuevo intervalo de incertidumbre cuando $\theta(\lambda)<\theta(\mu)$

La reducción del intervalo de incertidumbre se realiza, observando el valor de la función objetivo $\left|X_{f}\right|$, evaluada en los valores $\lambda$ y $\mu$.Si $\left|X_{f}(\lambda)\right|>\left|X_{f}(\mu)\right|$, entonces $\left|X_{f}(g)\right| \geq\left|X_{f}(\mu)\right|$, para todo 
$g \in[0, \lambda)$; por lo tanto, el valor mínimo de $\left|X_{f}\right|$, se encuentra en $[\lambda, b]$, que se define como el nuevo intervalo de incertidumbre. La Figura 5 , muestra la definición del nuevo intervalo de incertidumbre cuando $\left|X_{f}(\lambda)\right|>\left|X_{f}(\mu)\right|$.

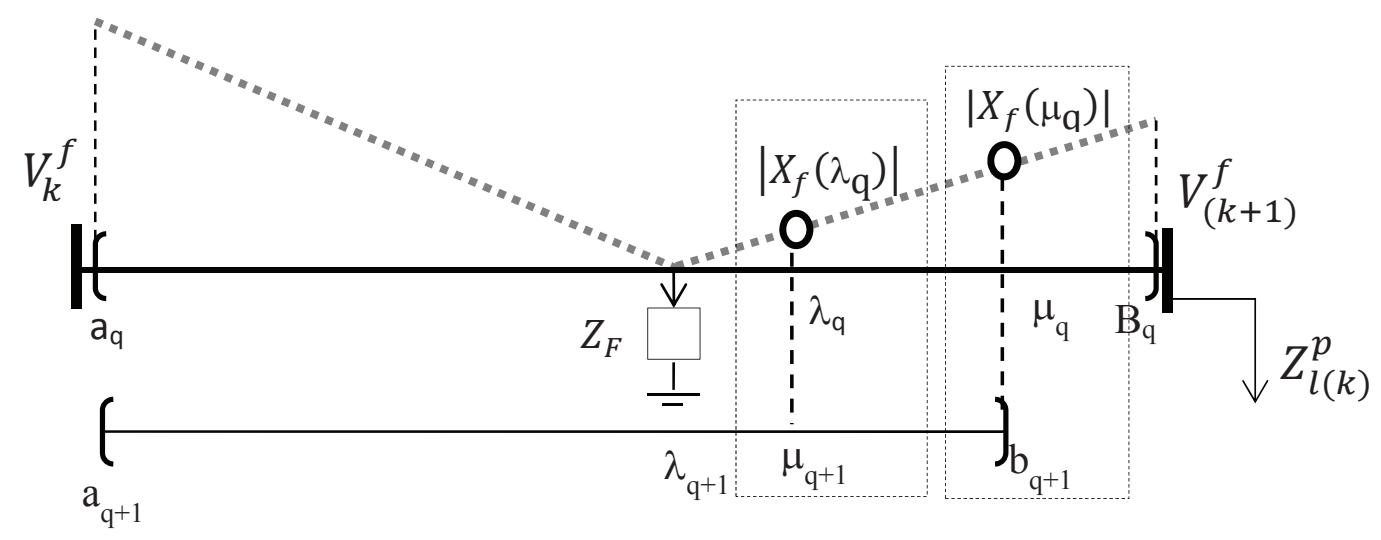

Figura 6. Determinación de nuevo intervalo de incertidumbre cuando $\theta(\lambda)<\theta(\mu)$ )

Si $\left|X_{f}(\lambda)\right|<\left|X_{f}(\mu)\right|$, entonces $\left|X_{f}(g)\right| \geq\left|X_{f}(\lambda)\right|$, para todo $g \in(\mu$, b]. Por lo tanto, el valor mínimo de $\left|X \_f\right|$, se encuentra en $[a, \mu]$, que se define como el nuevo intervalo de incertidumbre, tal como se muestra en la Figura 6.

Se puede observar que el intervalo de incertidumbre es cada vez más pequeño y el resultado final será un intervalo más pequeño que contiene el valor mínimo de la función $\left[a_{n}, b_{n}\right]$. El tamaño mínimo de este intervalo se define por medio de la tolerancia en la precisión del método, que a su vez, determina los números de la serie de Fibonacci por utilizar en la minimización de la función. Por lo tanto, si se desea una cierta tolerancia tol, la longitud del intervalo de incertidumbre $\left[a_{n}\right.$, $\left.b_{n}\right]$, debe cumplir con (17).

$$
b_{n}-a_{n} \leq \mathrm{tol}
$$

Como en cada iteración, el intervalo de incertidumbre se reduce en un valor $\Delta \mathrm{W}$ dado por la ecuación (18), y en la iteración ( $\mathrm{n}-1)$, se tiene un $\lambda_{n-1}=\mu_{n-1}$, se puede determinar una expresión para la longitud del intervalo final de incertidumbre $\left[a_{n}, b_{n}\right]$, en función de los números de Fibonacci, tal como se muestra en la formula (19).

$$
\Delta W=\left(W_{n-q} / W_{n-q+1}\right)
$$




$$
\begin{gathered}
b_{n}-a_{n}=\left(\frac{W_{n-1}}{W_{n}}\right) \times\left(\frac{W_{n-2}}{W_{n-1}}\right) \times\left(\frac{W_{n-3}}{W_{n-2}}\right) \ldots \ldots \ldots\left(\frac{W_{1}}{W_{2}}\right) \times\left(b_{1}-a_{1}\right) \\
b_{n}-a_{n}=\left(\frac{W_{1}}{W_{n}}\right) \times\left(b_{1}-a_{1}\right)
\end{gathered}
$$

Con $W_{1}=1$ y reemplazando $b_{n}-a_{n}$ en (17), se obtiene una expresión para el n-ésimo numero de Fibonacci en función de la tolerancia, tal como se muestra en (20) [6].

$$
W_{n} \geq \frac{b_{1}-a_{1}}{t o l}
$$

Como el resultado obtenido por la técnica de reducción de intervalos de incertidumbre de Fibonacci es un intervalo $\left[a_{n}, b_{n}\right]$, la distancia a la falla $\mathrm{m}$ se define en función de este intervalo como se muestra en la expresión (21).

$$
m=\frac{b_{n}+a_{n}}{2}
$$

Por último, en la Figura 7 se muestra la estructura básica del algoritmo implementado para la aplicación sistematizada de la estrategia de reducción de intervalos de Fibonacci, para estimar la distancia de falla en cada sección del sistema en estudio.

Como se demostró, esta estrategia de localización de fallas tiene una característica diferente asociada a la propuesta de un paso variable, ya que los métodos de localización antes propuestos y basados en el concepto de la mínima reactancia, utilizan un tamaño de paso constante para determinar la reactancia de falla $[3,4,5]$. En la estrategia propuesta, $m$ se sustituye por los parámetros $\lambda$ y $\mu$ que varían respecto a la secuencia de números de Fibonacci, lo cual permite que el tamaño de paso para graficar la reactancia de falla sea variable. Esto último permite concentrar la mayor cantidad de puntos cerca del punto mínimo de la función, permitiendo una mayor precisión en la localización de la falla. En la Figura 8, se muestra una gráfica de reactancia en la sección en falla por un método convencional y un método, utilizando la técnica de reducción de intervalos de Fibonacci. 


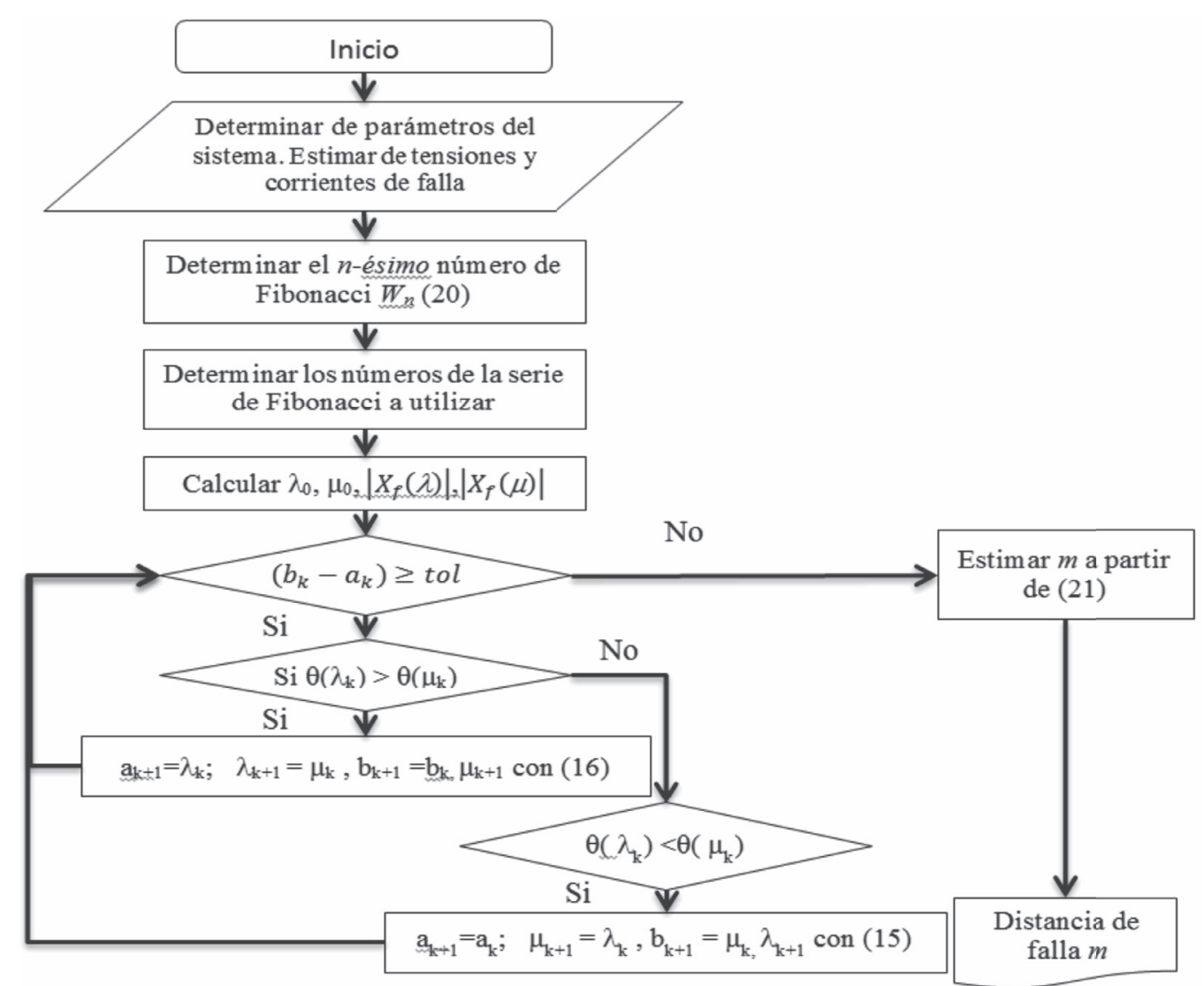

Figura 7. Algoritmo para estimar la distancia a la falla con el método de Fibonacci.
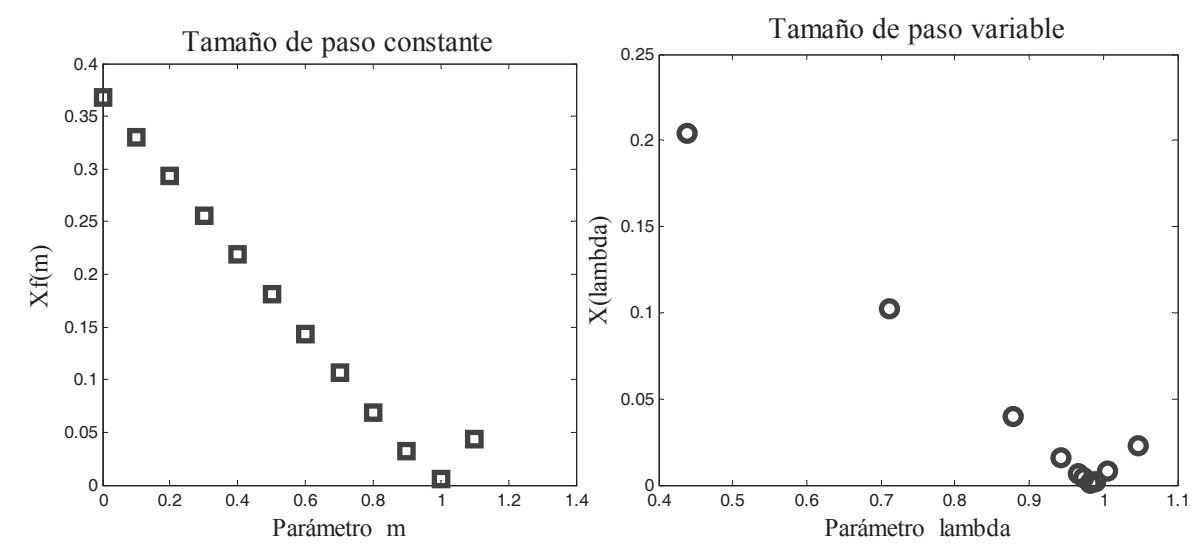

Figura 8. Gráfica de reactancia en la sección en falla por un método convencional y un método utilizando la técnica de reducción de intervalos de Fibonacci 


\section{ESTRATEGIA PROPUESTA}

La técnica mostrada en la sección 2, permite estimar la distancia a la falla, y por lo tanto, se debe desarrollar un algoritmo que permita generalizar este análisis para cada sección del sistema, actualizando todos los parámetros necesarios para estimar la reactancia de falla. La Figura 9, muestra el algoritmo generalizado para localizar fallas.

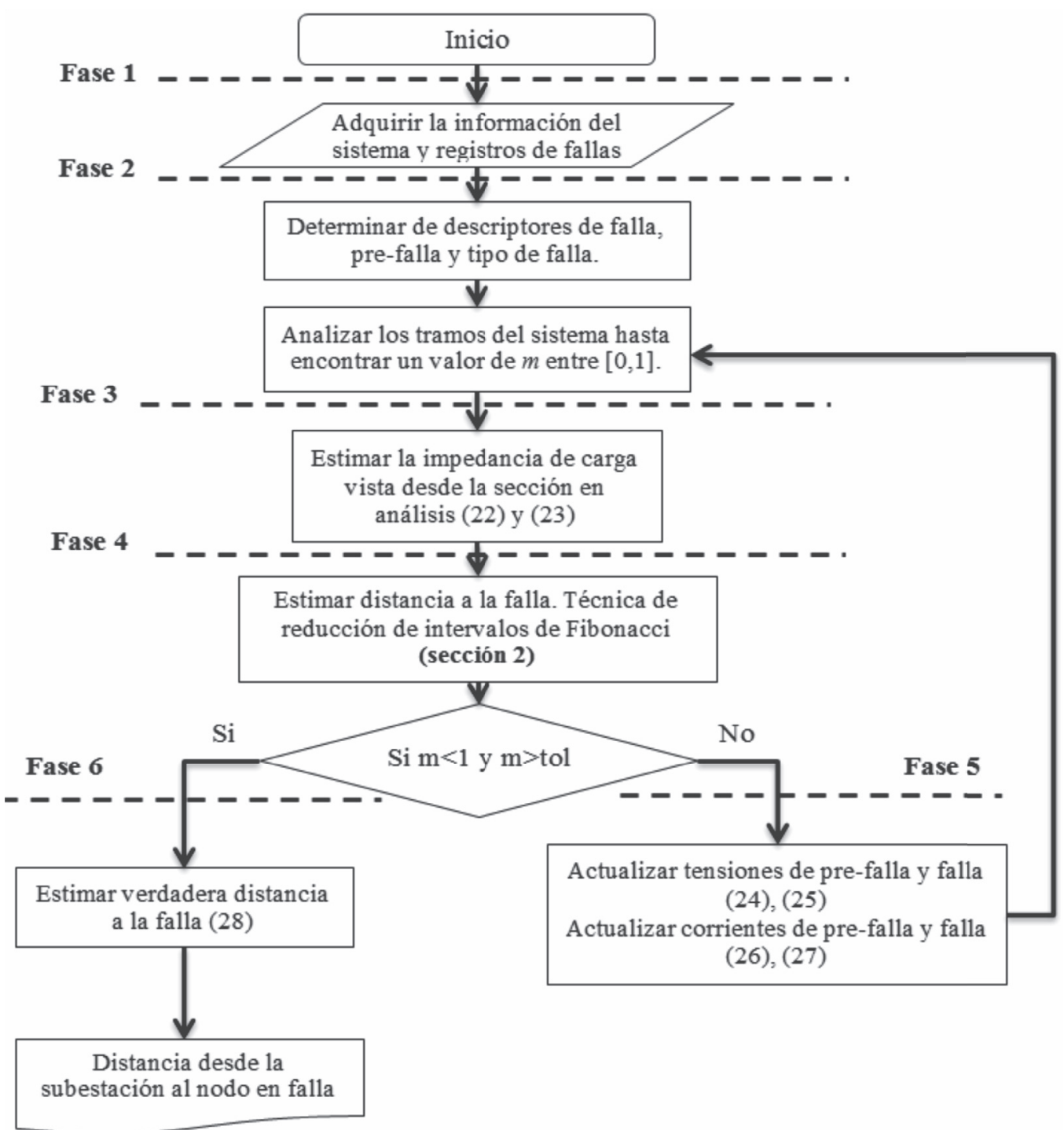

Figura 9. Algoritmo general de localización de fallas, utilizando reducción de intervalos de incertidumbre de Fibonacci

El algoritmo está compuesto por seis fases, que se explican en los numerales del 3.1 al 3.6. 


\subsection{FASE 1: INGRESO DE INFORMACIÓN DEL SISTEMA Y REGISTROS DE FALLAS}

La información del sistema se obtiene a partir de la simulación del sistema en estudio en el software EMPT/ATP. Asociado a EMPT/ATP, se utiliza una herramienta conocida como ATPeXchange que permite extraer, procesar y organizar la información del sistema y almacenarla en un formato legible para el software de programación Matlab [10].

\subsection{FASE 2: DETERMINACIÓN DE DESCRIPTORES DE PRE-FALLA, FALLA Y TIPO DE FALLA}

Los descriptores de falla y pre-falla corresponden a la caracterización de las señales del registro de falla a partir de los fasores de tensión y corriente en los estados de pre-falla y falla. Para validar el método, se utiliza una herramienta de simulación automática de fallas, que permite generar una base de datos de fallas de un sistema de potencia [8].Los descriptores utilizados para localizar la falla son la magnitud de tensión y corriente en los estados de pre-falla y falla, y sus respectivos ángulos de fase. El tipo de falla se determina por medio del algoritmo propuesto por Ratan Das [1 1].

\subsection{FASE 3: ESTIMACIÓN DE LA CARGA DEL SISTEMA VISTA EN CADA NODO DEL SISTEMA}

Si se analiza un sistema de distribución como el que se muestra en la Figura 10, a partir de las corrientes y tensiones en estado de pre-falla para cada nodo del sistema, se puede realizar un análisis en pre-falla para una sección determinada, reduciendo el circuito mostrado en la Figura 11.

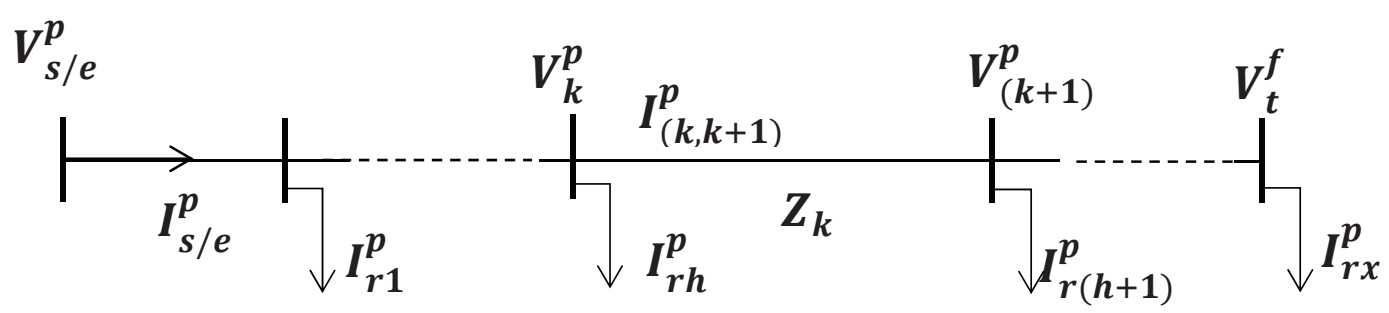

Figura 10. Sistema de distribución en estudio

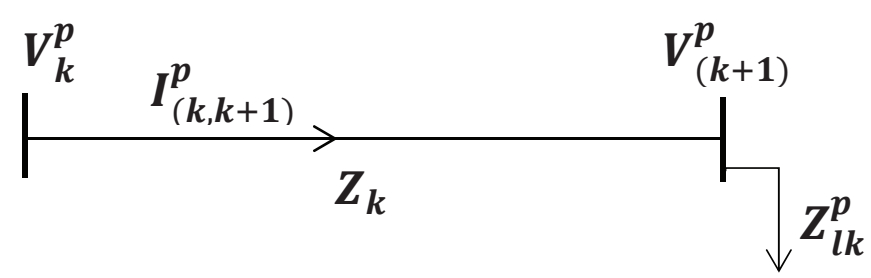

Figura 11 . Sistema de reducido 
Donde, las variables en estado de pre-falla indicadas con el superíndice p son:

$\boldsymbol{V}_{\boldsymbol{s}}^{\boldsymbol{p}} / \boldsymbol{e}$ : Tensión en la subestación

$\boldsymbol{I}_{\boldsymbol{s}}^{\boldsymbol{p}} \boldsymbol{\boldsymbol { p }} \boldsymbol{e}$ : Corriente en la subestación

$\boldsymbol{V}_{\boldsymbol{k}}^{\boldsymbol{p}}$ : Tensión en el nodo $\mathrm{k}$

$\boldsymbol{I}_{(\boldsymbol{k}, \boldsymbol{k}+\mathbf{1})}^{\boldsymbol{p}}$ : Corriente en la sección $(\mathrm{k}, \mathrm{k}+1)$

$\boldsymbol{I}_{\boldsymbol{r} \boldsymbol{h}}^{\boldsymbol{p}}$ : Corriente por el lateral $\mathrm{h}$

$\boldsymbol{Z}_{\boldsymbol{k}}$ : Impedancia de línea en la sección en análisis

$\boldsymbol{Z}_{\boldsymbol{l} \boldsymbol{k}}^{\boldsymbol{p}}$ : Impedancia de carga vista desde la sección en análisis

Como la impedancia de carga se estima con las mediciones de tensión y corriente de pre-falla, y la variación de la carga en el sistema es muy lenta en comparación con el cambio de estado de prefalla a falla, se puede asumir que la impedancia de carga en el instante de pre-falla, es la misma que en el estado de falla. Por lo tanto, la impedancia de carga vista en cada nodo del sistema de potencia, se define por el sistema de ecuaciones (22).

$$
\begin{aligned}
& Z_{l a_{k}}=\frac{V_{k_{a}}^{p}-\left(Z_{a a} \times I_{(k, k+1)_{a}}^{p}+Z_{a b} \times I_{(k, k+1)_{b}}^{p}+Z_{a c} \times I_{(k, k+1)_{c}}^{p}\right)}{I_{(k, k+1)_{a}}^{p}} \\
& Z_{l b_{k}}=\frac{V_{k_{b}}^{p}-\left(Z_{b a} \times I_{(k, k+1)_{a}}^{p}+Z_{b b} \times I_{(k, k+1)_{b}}^{p}+Z_{b c} \times I_{(k, k+1)_{c}}^{p}\right)}{I_{(k, k+1)_{b}}^{p}} \\
& Z_{l c_{k}}=\frac{V_{k_{c}}^{p}-\left(Z_{c a} \times I_{(k, k+1)_{a}}^{p}+Z_{c b} \times I_{(k, k+1)_{b}}^{p}+Z_{c c} \times I_{(k, k+1)_{c}}^{p}\right)}{I_{(k, k+1)_{c}}^{p}}
\end{aligned}
$$

De esta manera, la matriz de impedancia de carga vista desde el nodo k, se define por (23).

$$
Z_{l k}=\left[\begin{array}{ccc}
Z_{l a_{k}} & 0 & 0 \\
0 & Z_{l b_{k}} & 0 \\
0 & 0 & Z_{l c_{k}}
\end{array}\right]
$$

\subsection{FASE 4: ESTIMACIÓN DE LA DISTANCIA A LA FALLA EN CADA SECCIÓN}

La estimación de la distancia de falla en cada sección, se hace por medio de la técnica de reducción de intervalos de incertidumbre de Fibonacci, de la manera propuesta en la sección 2. 


\subsection{FASE 5: ACTUALIZACIÓN DE CORRIENTES Y TENSIONES DE FALLA Y PRE-FALLA}

La actualización de tensiones y corrientes de falla y pre-falla corresponde al cálculo de la tensión de recibo en la sección actual que a su vez, es la tensión de envío en la siguiente sección. Este cálculo se realiza por medio de las expresiones (24) y (25), respectivamente.

$$
\begin{aligned}
& \mathrm{V}_{(\mathrm{k}+1)}^{\mathrm{p}}=\mathrm{V}_{(\mathrm{k})}^{\mathrm{p}}-\mathrm{Z}_{\mathrm{k}} \times \mathrm{I}_{(\mathrm{k}, \mathrm{k}+1)}^{\mathrm{p}} \\
& \mathrm{V}_{(\mathrm{k}+1)}^{\mathrm{f}}=\mathrm{V}_{(\mathrm{k})}^{\mathrm{f}}-\mathrm{Z}_{\mathrm{k}} \times \mathrm{I}_{(\mathrm{k}, \mathrm{k}+1)}^{\mathrm{f}}
\end{aligned}
$$

La actualización de la corriente se realiza, utilizando la admitancia nominal por cada lateral, donde se calcula la corriente para la siguiente sección, utilizando los valores de tensión y corriente de la sección actual, como se muestra en las ecuaciones (26) y (27).

$$
\begin{aligned}
& \mathrm{I}_{(\mathrm{k}+1, \mathrm{k}+2)}^{\mathrm{p}}=\mathrm{I}_{(\mathrm{k}, \mathrm{k}+1)}^{\mathrm{p}}-\mathrm{Y}_{\text {ramalk }} \times \mathrm{V}_{(\mathrm{k}+1)}^{\mathrm{p}} \\
& \mathrm{I}_{(\mathrm{k}+1, \mathrm{k}+2)}^{\mathrm{f}}=\mathrm{I}_{(\mathrm{k}, \mathrm{k}+1)}^{\mathrm{f}}-\mathrm{Y}_{\text {ramalk }} \times \mathrm{V}_{(\mathrm{k}+1)}^{\mathrm{f}}
\end{aligned}
$$

\subsection{FASE 6: ESTIMACIÓN DE LA DISTANCIA REAL DESDE LA SUBESTACIÓN AL NODO DE FALLA}

La estimación de la distancia real al nodo en falla, se realiza por medio de la sumatoria de las distancias de las secciones recorridas por el algoritmo, adicionándole la porción de la sección donde se localiza la falla. La ecuación (28), define el valor de la distancia de falla estimada.

$$
L_{e s t}=\sum_{i=1}^{n t-1}\left(L_{\text {tramo }_{i}}\right)+m_{\text {último }} \times L_{\text {tramo }_{n t}}
$$

Donde:

$L_{\text {est }}$ : Distancia estimada desde la subestación al punto donde se localiza la falla.

nt: Número de tramos analizados

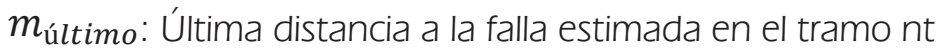

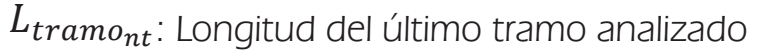

\section{PRUEBAS Y RESULTADOS}

El sistema seleccionado para realizar las pruebas y validación de la estrategia propuesta es el sistema IEEE 34 nodos [12]. Este alimentador está ubicado en el estado de Arizona (Estados Unidos), y opera a una tensión de 24.9 kV. Entre sus principales características, están la presencia de laterales monofásicos, bifásicos y trifásicos, múltiples calibres de conductor, cargas desbalanceadas de 
naturaleza concentrada y distribuida. Este sistema se modela en el software de simulación ATPDraw, con el cual se simularon fallas monofásicas, bifásicas y trifásicas sobre un rango de resistencias de falla de 0 a 40 ohms. La Figura 12 muestra el sistema IEEE34 en el entorno del ATP Draw [13].

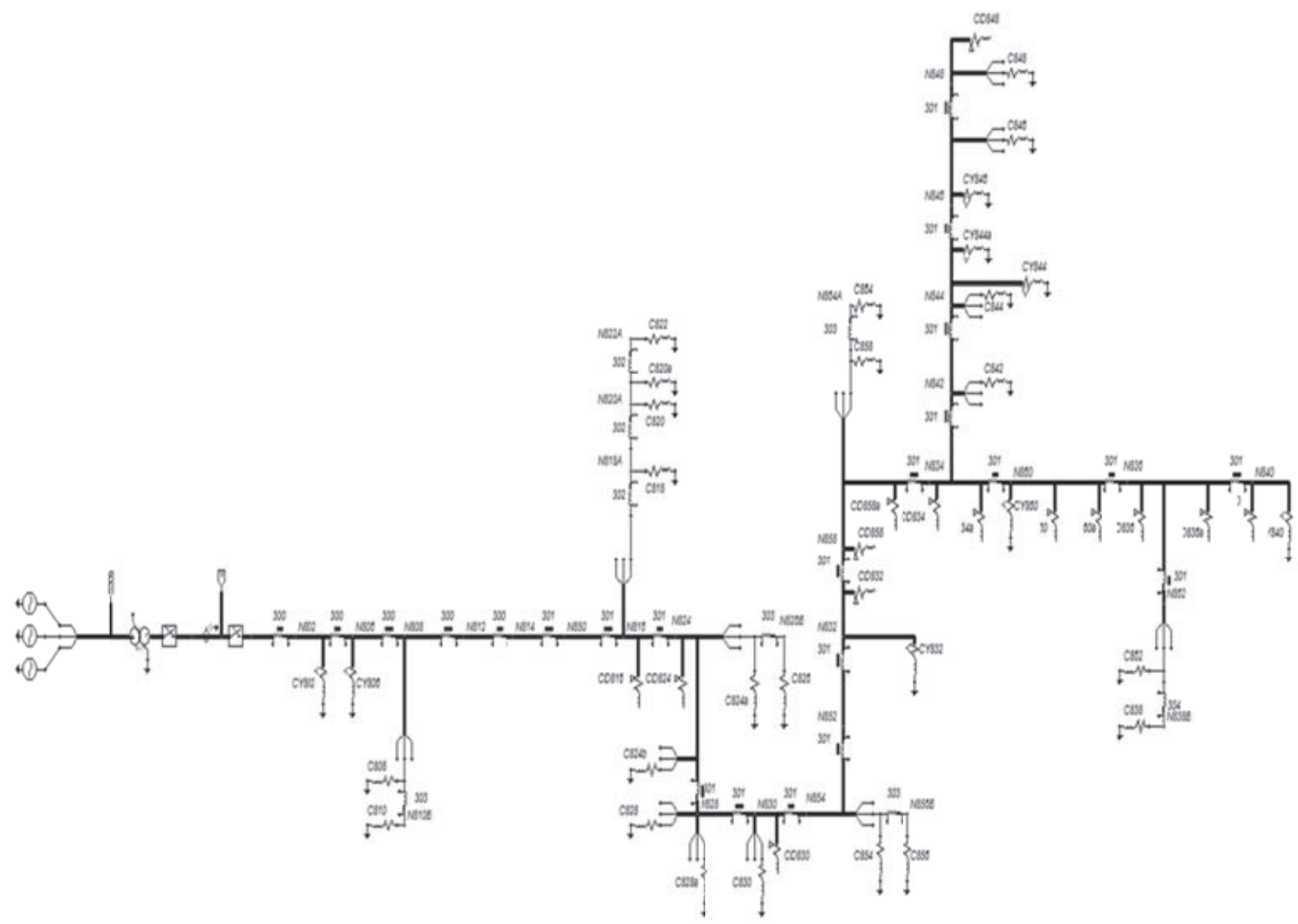

Figura 12. Sistema IEEE 34 en ATP Draw

Los resultados se muestran a partir de las gráficas de desempeño del método, donde se comparan el error de la distancia calculada y la distancia real de la falla. El error propuesto se calcula a partir de la ecuación (29).

$$
\text { Error }=\frac{\left|d_{\text {estimada }}-d_{\text {real }}\right|}{d_{\text {total }}}
$$

Los resultados para las fallas monofásicas se presentan en la Figura 13. Para este tipo de falla, el error es menor en el inicio y presenta una tendencia creciente. También, se puede concluir a partir la gráfica de desempeño, que el valor de la resistencia de falla impacta directamente en la precisión de la localización, donde el mayor error presente para la falla monofásica es cercano al 1,5\%. 


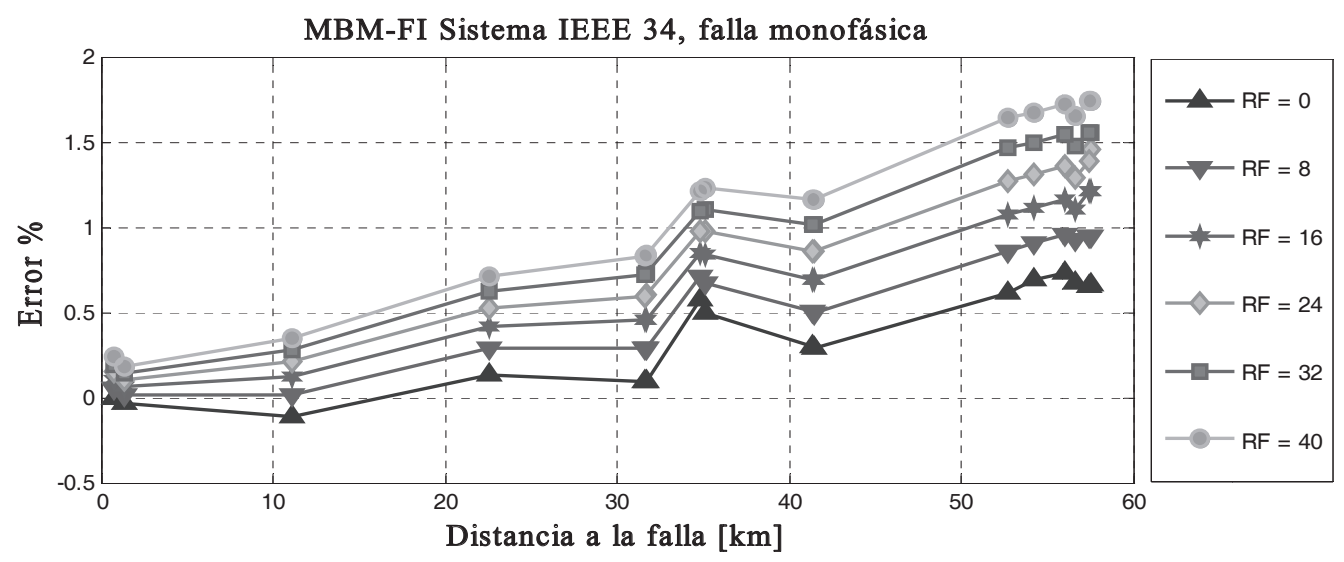

Figura 13. Curvas de desempeño, falla monofásica.

Otra característica que afecta directamente el desempeño del método, es el desbalance de carga. Por esta razón, se realizan pruebas en un sistema que presente diferentes tipos de líneas (monofásicas, bifásicas y trifásicas), de tal manera que el circuito se aproxime a un sistema real.

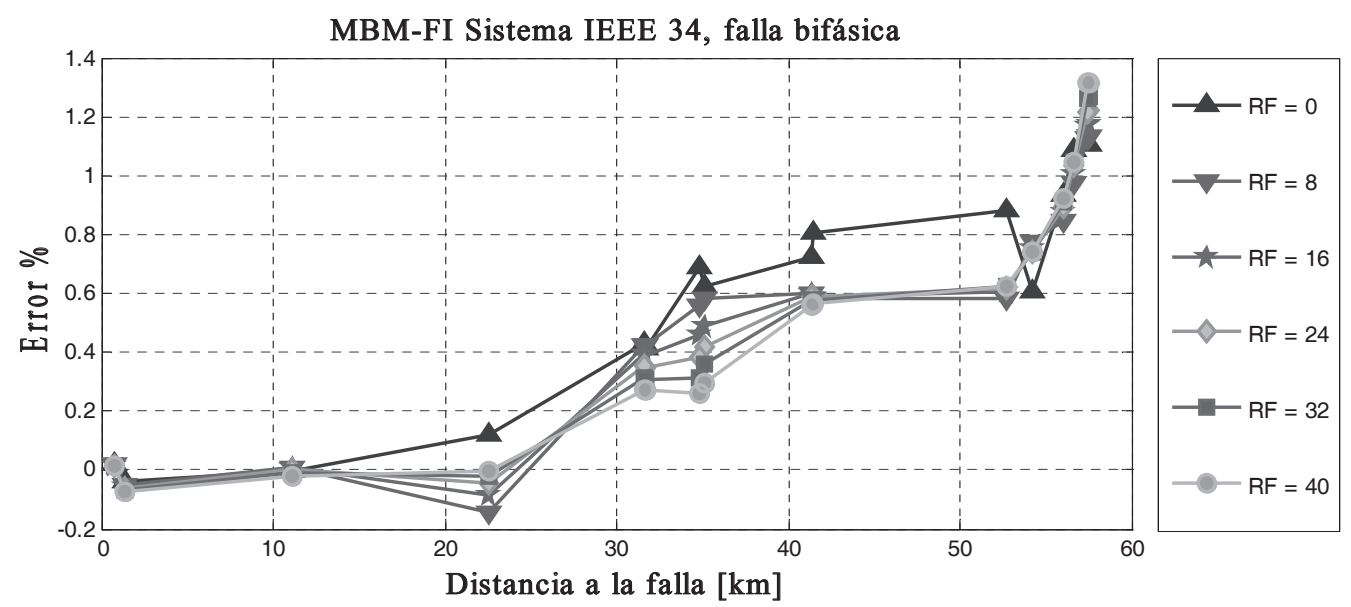

Figura 14. Curvas de desempeño, falla bifásica.

En la Figura 14, se presentan los resultados para las fallas bifásicas, donde se muestran las curvas de desempeño error contra distancia real.

La estrategia de localización propuesta presenta un comportamiento de sobrestimación de la distancia a la falla. Este comportamiento se presenta debido a la incertidumbre que se tiene sobre el verdadero valor de la carga. No obstante, su desempeño es sobresaliente, pues presenta un error en la precisión, de cerca de 0,8\%. 
Por último, la Figura 15 presenta los resultados para las fallas trifásicas, donde se muestran las curvas de desempeño de error contra distancia real.

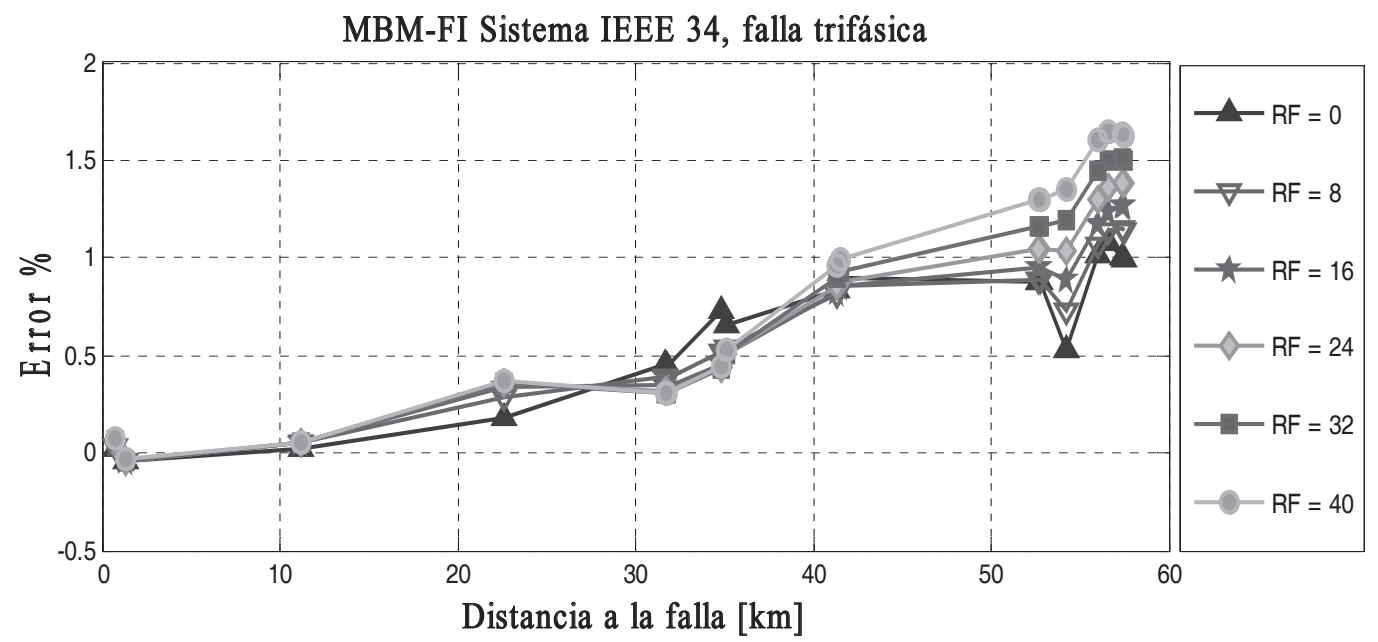

Figura 15. Curvas de desempeño, falla trifásica

El mejor desempeño de la estrategia se presenta para la falla bifásica, con un error cercano a 0,8\%. Para las fallas monofásica y trifásica, su desempeño es muy similar aunque en la falla monofásica, se nota una clara diferenciación entre las curvas con diferentes resistencias de falla. No obstante, el error máximo de validación para ambos tipos de falla es aceptable y no representa una gran incertidumbre en la localización de la falla.

\section{CONCLUSIONES}

La estrategia propuesta para localizar fallas paralelas en sistemas de distribución, permite determinar de manera precisa el punto donde ocurrió la falla, a partir de las mediciones de tensión y corriente en los estados de pre-falla y falla en la subestación. Considerando que sólo requiere las mediciones de tensión y corriente y la configuración del sistema, esta estrategia es de fácil implementación en un sistema de potencia real.

Por otra parte, la utilización de la técnica de reducción de intervalos de Fibonacci para desarrollar una estrategia de localización de fallas basada en el principio de la mínima reactancia, permite generar una estrategia de búsqueda que concentra su esfuerzo muy cerca del mínimo de la función definida por la reactancia de falla, lo cual mejora su precisión. Para el caso de prueba propuesto, se aprecia que los errores en la estimación de la distancia a la falla son siempre inferiores al $2 \%$ en los tipos de fallas monofásicas, bifásicas y trifásicas, considerando resistencias de falla de hasta 40 ohms.Además, la estimación de la impedancia vista desde la sección en análisis, a 
partir de las mediciones de tensión y corriente registradas en la subestación permite considerar cualquier variación de carga, lo cual mejora considerablemente el desempeño de la metodología.

Por último, si se utiliza una estrategia de estimación de la distancia de falla para cada sección del sistema, permite actualizar sus tensiones y corrientes de pre-falla y falla. Este proceso mejora considerablemente la precisión de la estrategia de localización de fallas.

\section{REFERENCIAS BIBLIOGRÁFICAS}

[1] Philipson L., and Lee Willis H., (2009). Understanding electric utilities and regulations. CRC Press. 2 Ed.

[2] Comisión de Regulación de Energía y Gas, (2008). Resolución CREG 097. Ministerio de minas y energía. Bogotá, pp. 1-135.

[3] Morales-España G., Mora-Flórez J., y Pérez-Londoño S., (2007). Método de localización de fallas en sistemas de distribución basado en gráficas de reactancia. En: Scientia et Technica. Vol. 34, pp. 49-54.

[4] Morales-España G., Mora-Flórez J., and Vargas-Torres H., (2010). Fault location method based on the determination of the minimum fault reactance for uncertainty loaded and unbalanced power distribution systems. In: Transmission and Distribution Conference and Exposition: Latin America (T\&D-LA), 2010 IEEE/PES.

[5] Correa-Tapasco E., Mora-Flórez J., and Pérez Londoño S., (2011). Generalized strategy for implementing the minimum fault reactance based fault location algorithm in real power distribution systems". In: Revista Ingeniería e Investigación, Vol. 31 (2), pp 71-75.

[6] Bazaraa Mokhtar S., (2006). Nonlinear programming: theory and algorithms. John Wiley \& Sons, $3^{\mathrm{a}}$ ed. West Sussex., Inglaterra.

[7] Morales G., Mora J., and Vargas H., (2009). Elimination of Multiple Estimation for Fault Location in Radial Power Systems by Using Fundamental Single-End Measurements. In: IEEE Transactions on power delivery, Vol. 24, (3).

[8] Pérez Hernández L., Mora-Flórez J., and Perez Londoño S., (2010). Design of an efficient and automated fault simulation tool applied to electric power systems. In: Revista Dyna. 164, pp. 178-188. 
[9] Bertsekas D., Nedic A., and Ozdaglar A., (2003). Convex Analysis and Optimization. Athena Scientific, Belmont, Massachusetts. pp. 20-35.

[10] Bedoya A., Mora Flórez J., and Pérez Londoño S., (2012). Estrategia de reducción para la aplicación generalizada de localizadores de fallas en sistemas de distribución de energía eléctrica. En: Revista EIA. N.17, pp. 21-37.

[1 1] Ratan Das, (1998). Determining the locations of faults in distribution systems. Thesis. (Ph.D. in Engineering). University of Saskatchewan Saskatoon.

[12] IEEE (2000). Distribution System Analysis Subcommittee.Radial Test Feeders. En:http://mww. ewh.iee.org/soc/pes/sacom/testfeeders .html. (diciembre 2011 ).

[13] Comité Argentino de Usuarios del EMTP/ATP_CAUE (2002). ATP.Alternative transient program rulebook. 